\title{
TRANSITION METAL IMPURITIES AND ELECTRONIC STRUCTURE OF ZnSe-BASED ISOVALENT SEMICONDUCTOR ALLOYS
}

\author{
T.P. Surkova ${ }^{a}$, W. Giriat ${ }^{b}$, M. Godlewski ${ }^{c}$ AND S. Permogorov ${ }^{d}$ \\ ${ }^{a}$ Institute of Metal Physics, Ural Division of Russian Academy of Sciences \\ 620219 Ekaterinburg GSP-170, Russia \\ ${ }^{b}$ IVIC, Centro de Fisica, Apto 1827, Caracas 1010A, Venezuela \\ 'Institute of Physics, Polish Academy of Sciences \\ Al. Lotników 32/46, 02-668 Warszawa, Poland \\ d A.F. Ioffe Physical-Technical Institute, Russian Academy of Sciences \\ 194021 St. Petersburg, Russia
}

\begin{abstract}
Energy level positions of the nickel $2+/ 1+$ and cobalt $2+/ 3+$ charge states have been used to estimate band edges for the valence and conduction bands of $\mathrm{ZnSe}$-based alloys with cation ( $\mathrm{ZnCdSe}$ ) and anion ( $\mathrm{ZnSSe}$ ) substitution. Chemical trends in band offsets of heterostructures of $\mathrm{Zn}$ - or Mn-based II-VI compounds are analysed. Further on, the change of $\mathrm{Ni}^{2+}\left(3 d^{8}\right)$ and $\mathrm{Co}^{2+}\left(3 d^{7}\right)$ intra- $d$ shell transition bands upon the alloying of host material is discussed.
\end{abstract}

PACS numbers: $71.55 . \mathrm{Gs}, 78.20 . \mathrm{Wc}$

Properties of transition metal impurities in binary semiconducting compounds have been intensively studied and are, in the most cases, well understood. It has been demonstrated that $3 d$ transition metal impurities are perfect "probes" for the study of compositional dependence of some fundamental characteristics of the electronic structure of semiconducting alloys, in particular, the respective band offsets [1-4] and of structural and compositional disordering. In our previous works [5-7] we undertook optical study of $\mathrm{ZnMnSe}, \mathrm{ZnCdSe}$ and $\mathrm{ZnSSe}$ alloys doped with $3 d$ transition metal ions to determine the energy level positions of the nickel $2+/ 1+$ and cobalt $2+/ 3+$ charge states through the wide composition range of the alloys. We derived respective band offsets as the energy difference of photoionization transitions. In this report we present new experimental data and discuss chemical trends in band offsets and behaviour of intra- $d$ shell transition bands upon the alloying.

The single crystals $\mathrm{Zn}_{1-x} \mathrm{Cd}_{x}$ Se:Co and $\mathrm{ZnS}_{x} \mathrm{Se}_{1-x}$ :Co were grown by chemical transport method and doped with Co to the level $10^{18} \mathrm{~cm}^{-3}$, approximately. Content of $\mathrm{Cd}(x=0 \div 0.5)$ and $\mathrm{S}(x=0 \div 1.0)$ was controlled by EDXRF 

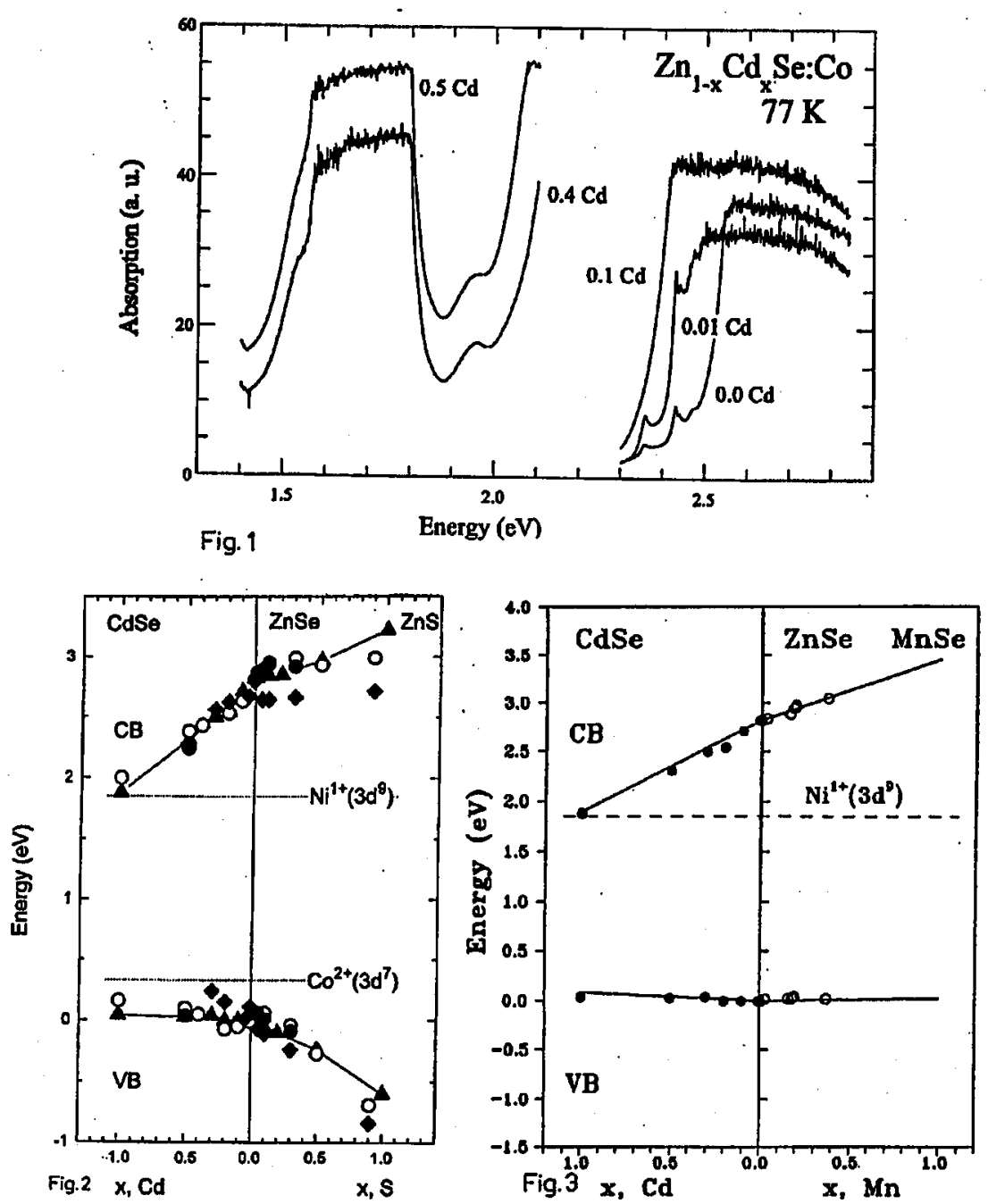

Fig. 1. Absorption spectra of $\mathrm{Zn}_{1-x} \mathrm{Cd}_{x}$ Se:Co near the photoionization threshold of the $\mathrm{Co}^{2+} \rightarrow \mathrm{Co}^{3+}$ and intra-shell ${ }^{4} A_{2}(F) \rightarrow{ }^{4} T_{1}(P)$ transitions for different alloy composition at $T=77 \mathrm{~K}$. Details of the absorption spectrum between $1.5-1.8 \mathrm{eV}$ and $2.5-2.9 \mathrm{eV}$ could not be resolved due to too strong absorption.

Fig. 2. Valence band offsets of $\mathrm{Zn}_{1-x} \mathrm{Cd}_{x} \mathrm{Se} / \mathrm{ZnSe}$ and $\mathrm{ZnS}_{x} \mathrm{Se}_{1-x} / \mathrm{ZnSe}$ measured relative to the $\mathrm{Ni}$ acceptor (filled $\Delta$ ) and $\mathrm{Co}(\circ, \bullet)$ donor levels for different temperatures: filled $\Delta, \circ-2 \mathrm{~K}, \bullet-77 \mathrm{~K}$, filled $\diamond-300 \mathrm{~K}$. The solid line is extrapolated from the TM averaging procedure for the corresponding binary compounds [4]. The value $E_{c}-1.7 \mathrm{eV}$ for Co donor level in CdSe was taken from Ref. [9]. The data for $300 \mathrm{~K}$ are shifted down in the energy, which reflects a decrease in the fundamental band gap energies.

Fig. 3. Valence band offsets of $\mathrm{ZnMn}_{x} \mathrm{Se}_{1-x} / \mathrm{ZnSe}$ measured relative to $\mathrm{Ni}$ acceptor level for $T=2 \mathrm{~K}$. The data are taken from Ref. [5]. 
analysis, also. Absorption spectra were recorded by DFS-12 and Hitachi 340 spectrometers. Measurements were done at 2, 77 and $300 \mathrm{~K}$. The observation of broad strong ionization absorption band in the range of 2.0-3.0 eV (Fig. 1) allowed us to estimate energy level positions of cobalt $2+/ 3+$ charge state [8]. Band gap energies were determined either from exciton seflectivity spectra or calculated from the well-established $E_{\mathrm{g}}(x)$ dependences. It was found that spectral dependence $\alpha\left(\hbar \omega-\hbar \omega_{\text {th }}\right)^{\beta}$ describes well the spectra, when $\beta$ instead of $1 / 2$ (allowed process) or $3 / 2$ (parity forbidden process) has some intermediate value, close to 1 . It can be connected with a perturbation of the photoionization band by intra- $d$ shell $\mathrm{Co}^{2+}$ transitions. The results of optical investigations are presented in Table and Fig. 2, together with our previous data on positions of valence and conduction band edges, measured relative to $\mathrm{Ni} 2+/ 1+$ acceptor level.

TABLE

Composition $x$, energy gap $E_{\mathrm{g}}$ and a threshold energy $\hbar \omega_{\mathrm{th}}$ for the Co photoionization $(2+\rightarrow 3+)$ in $\mathrm{Zn}_{1-x} \mathrm{Cd}_{x} \mathrm{Se}$ :Co and $\mathrm{ZnS}_{x} \mathrm{Se}_{1-x}$ :Co alloys at $2 \mathrm{~K}$.

\begin{tabular}{l|l|c|c}
\hline \hline Compound & $x$ & $E_{\mathrm{g}}[\mathrm{eV}]$ & $\hbar \omega_{\text {th }}[\mathrm{eV}]$ \\
\hline $\mathrm{ZnSe:Co}$ & 0.0 & 2.82 & 2.49 \\
\hline & 0.01 & 2.81 & 2.48 \\
& 0.1 & 2.68 & 2.44 \\
$\mathrm{Zn}_{1-x} \mathrm{Cd}_{x} \mathrm{Se}: \mathrm{Co}$ & 0.2 & 2.60 & 2.2 \\
& 0.4 & 2.38 & 2.1 \\
& 0.5 & 2.34 & 2.05 \\
\hline & 0.05 & 2.84 & 2.56 \\
& 0.1 & 2.88 & 2.61 \\
$\mathrm{ZnS}_{x} \mathrm{Se}_{1-x}: \mathrm{Co}$ & 0.3 & 3.03 & 2.66 \\
& 0.5 & 3.20 & 2.60 \\
& 0.9 & 3.68 & 2.66 \\
& 1.0 & 3.82 & 2.66
\end{tabular}

One can see that for CdSe/ZnSe values of band offsets obtained from the energies both $\mathrm{Ni} 2+/ 1+$ and $\mathrm{Co} 2+/ 3+$ photoionization transitions are very close to each other and for pure CdSe/ZnSe give the valence band offsets $(0.1 \pm 0.005) \mathrm{eV}$. It is near the values $(0.13 \pm 0.07) \mathrm{eV}$ and $(0.17 \pm 0.01) \mathrm{eV}$ determined by other methods [10], and predicted by the common anion rule. Previously obtained values of band offsets for another compound with cation substitution $\mathrm{ZnMnSe} / \mathrm{ZnSe}$ [5] also demonstrate good agreement of our results (Fig. 3) with both theoretical calculations [11] and independent experimental data [12].

For compounds with anion substitution $\mathrm{ZnSe} / \mathrm{ZnSSe}$ we got different values for the conduction band offset from Co $2+/ 3+$ ionization energies, and from ones of $\mathrm{Ni} 2+/ 1+$. At the first case it is equal to $(0.17 \pm 0.05) \mathrm{eV}$, and close to the 
value 0.2 , calculated by Harrison, and a number of experimental data [12]. From another side, a more pronounced shift of the conduction band $\mathrm{ZnSe} / \mathrm{ZnSSe}$, derived from $\mathrm{Ni} 2+/ 1+$ photoionization energies, is relatively close to those obtained from the simple electron affinity rule [5], averaging procedure [4] and theoretical calculations [10].

Infra-shell transitions of $\mathrm{Co}^{2+}\left(3 d^{7}\right)$ are also modified with the change of the alloy composition (Fig. 1). We controlled changes with composition and temperature of three groups of transitions: ${ }^{4} A_{2}(F) \rightarrow{ }^{4} T_{1}(F),{ }^{4} A_{2}(F) \rightarrow{ }^{4} T_{1}(P)$ and $L$, $M$ and $N$ lines at the Co photoionization threshold. All these bands show weak dependence on temperature. The effect of alloying on broadering and line shift is pronounced for $L, M$ and $N$-lines, only. Their behaviour with an increase in $\mathrm{Cd}$ or $\mathrm{S}$ content is close to that of $\mathrm{Ni}^{2+}{ }^{3} T_{1}(F) \rightarrow{ }^{3} T_{2}(F)$ and ${ }^{3} T_{1}(F) \rightarrow T_{2}(G)$ transitions.

Concluding, we have determined energy level position of Co $2+/ 3+$ charge state for a wide range of $\mathrm{Cd}$ and $\mathrm{S}$ fractions and temperatures in $\mathrm{Zn}_{1-x} \mathrm{Cd}_{x} \mathrm{Se}$ and $\mathrm{ZnS}_{x} \mathrm{Se}_{1-x}$ alloys. The estimated band edges for the valence and conduction bands are in relatively good agreement with our previous data, obtained from $\mathrm{Ni}$ $2+/ 1+$ energy level position $[5,6]$, the theory and a number of experimental data for corresponding superlattices [12].

This work has been partly supported by the RFBR (grant N 96-02-16496) and INTAS (grant N 93-3657).

\section{References}

[1] A. Zunger, Phys. Rev. Lett. 54, 848 (1985).

[2] M.J. Caldas, A. Fazzio, A. Zunger, Appl. Phys. Lett. 45, 671 (1984).

[3] J.M. Langer, H. Heinrich, Phys. Rev. Lett. 55, 1414 (1985).

[4] J.M. Langer, C. Delerue, M. Lannoo, H. Ileinrich, Phys. Rev. B 38, 7723 (1988).

[5] V.R. Galakhov, T.P. Surkova, V.I. Sokolov, E.Z. Kurmaev, Chr. Zubragel, H. Unlu, M. Neumann, S.A. Permogorov, L.N. Tenishev, Solid State Commun. 94, 279 (1994).

[6] T. Surkova, W. Giriat, M. Godlewski, P. Kaczor, S. Permogorov, L. Tenishev, J. Cryst. Growth 159, 471 (1996).

[7] T.P. Surkova, W. Giriat, S.A. Permogorov, L.N. Tenishev, M. Godlewski, P. Kaczor, A. Zakrzewski, in: Proc. 23 Int. Conf. on Physics of Semiconductors, Eds. M. Sceffler, R. Zimmermann, World Scientific, Singapore 1996, p. 2981.

[8] J.M. Noras, H.R. Szawelska, J.W. Allen, J. Phys. C, Solid State Phys. 14, 2355 (1981).

[9] J.M. Baranowski, An. Phuong, Phys. Status Solidi B 122, 331 (1984).

[10] S. Lankes, T. Reisinger, B. Hahn, C. Meier, M. Meier, W. Gebhardt, J. Cryst. Growth 159, 480 (1996).

[11] Su-Huai Wei, A. Zunger, Phys. Rev. B 53, R10457 (1996).

[12] P.J. Parbrook, K.P. O'Donnell, in: Optical Properties of Wide Bandgap II-VI Superlattices, in II-VI Semiconducting Compounds, Ed. M. Jain, World Scientific, Singapore 1993, p. 255. 